Systems Engineering Group

Department of Mechanical Engineering

Eindhoven University of Technology

PO Box 513

5600 MB Eindhoven

The Netherlands

http://seweb.se.wtb.tue.nl/
SE-Report: Nr. 2006-01

\section{Modeling and Analysis of Manufacturing Systems}

E. Lefeber and J.E. Rooda 


\section{Chapter 1}

\section{Modeling and Analysis of Manufacturing Systems}

\subsection{Introduction}

The dynamics of manufacturing systems has been a subject of study for several decades (Forrester 1961; Hopp and Spearman 2000). Over the last years, manufacturing systems have become more and more complex. A good understanding of the dynamics of manufacturing systems has therefore become even more important.

The goal of this chapter is to introduce a large variety of models that have been used for modeling manufacturing systems. By means of examples it is illustrated how certain modeling techniques can be used to derive models that can be used for analysis and/or control. Also, references are provided that can be used as a starting point for getting more details.

Furthermore, recent developments in the modeling, analysis and control of manufacturing systems are presented.

Since no familiarity with manufacturing systems is assumed, in Section 1.2 some terminology and basic properties of manufacturing systems are introduced. Section 1.3 provides some analytical modeling techniques and methods for analyzing steady state behavior of manufacturing systems. Section 1.4 is concerned with deriving discrete event models of manufacturing systems, which yield a more detailed insight in the dynamics of a manufacturing system. In order to reduce the complexity of discrete event models, effective proces times (EPT's) are introduced in Section 1.5, which results in modeling a manufacturing system as a large queueing network. This way of modeling a manufacturing system is a first step in a larger control framework which is introduced in Section 1.6. This control framework makes it possible to study problems on controlling the dynamics of manufacturing systems by means of the available inputs. An important role in this control framework is played by approximation models. The most commonly used approximation models are presented in Section 1.7. Recently a new class of approximation models has been proposed which is presented in Section 1.8. Section 1.9 concludes this chapter.

\subsection{Preliminaries}

First a few basic quantities are introduced as well as the main principles for manufacturing system analysis. The items produced by a manufacturing system are called lots. Also the words product and job are commonly used. Other important notions are throughput, flow time, wip and 


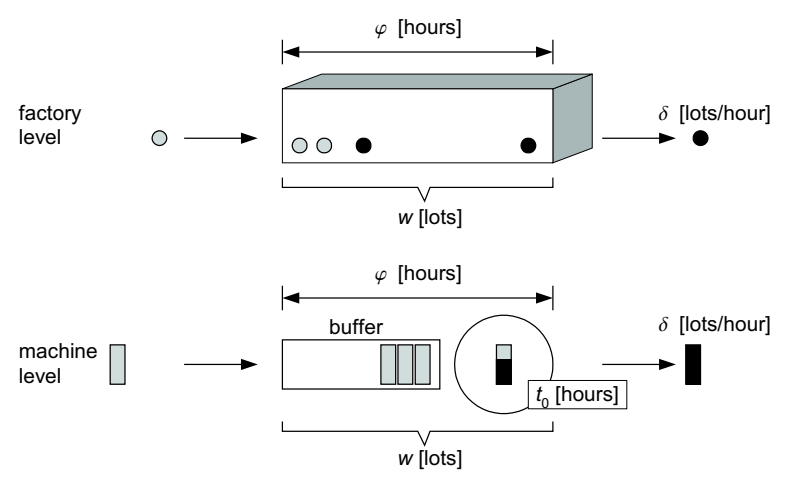

Figure 1.1: Basic quantities for manufacturing systems

utilization. These notions are illustrated in Figure 1.1 at factory and machine level.

Raw process time $t_{0}$ denotes the net time a lot needs processing on a machine. This process time excludes additions such as setup time, breakdown, or other sources that may increase the time a lot is on the machine. The raw process time is typically measured in hours or minutes.

Throughput $\delta$ denotes the number of lots per time-unit that leaves the manufacturing system. At machine level, this denotes the number of lots that leave a machine per time-unit. At factory level it denotes the number of lots that leave the factory per time-unit. The unit of throughput is typically lots/hour.

Flow time $\varphi$ denotes the time a lot is in the manufacturing system. At factory level this is the time from release of the lot in the factory until the finished lot leaves the factory. At machine level this is the time from entering the machine (or the buffer in front of the machine) until leaving the machine. Flow time is typically measured in days, hours, or minutes. Instead of flow time the words cycle time and throughput time are also commonly used.

Work in process (wip) $w$ denotes the total number of lots in the manufacturing system, i.e. in the factory or in the machine. Wip is measured in lots.

Utilization $u$ denotes the fraction a machine is not idle. A machine is considered idle if it could start processing a new lot. Thus process time as well as downtime, setup-time and preventive maintenance time all contribute to the utilization. Utilization has no dimension. Utilization can never exceed 1.0.

Ideally, a manufacturing system should both have a high throughput and a low flow time or low wip. Unfortunately, these goals can not both be met simultaneously. These two goals are conflicting, as can be seen from Figure 1.2. On the one hand, if a high throughput is required, machines should always be busy. As from time to time disturbances like machine failures happen, buffers between two consecutive machines are required to make sure that the second machine can still continue if the first machine fails (or vice versa). Therefore, for a high throughput many lots are needed in the manufacturing system, i.e. wip needs to be high. As a result, if a new lot starts in the system it has a large flow time, since all lots that are currently in the system need to be completed first.

On the other hand, the least possible flow time can be achieved if a lot arrives at a completely empty system and never has to wait before processing at any machine takes place. As a result, for that system the wip level is small, but also most of the time machines are not processing, yielding a small throughput. 

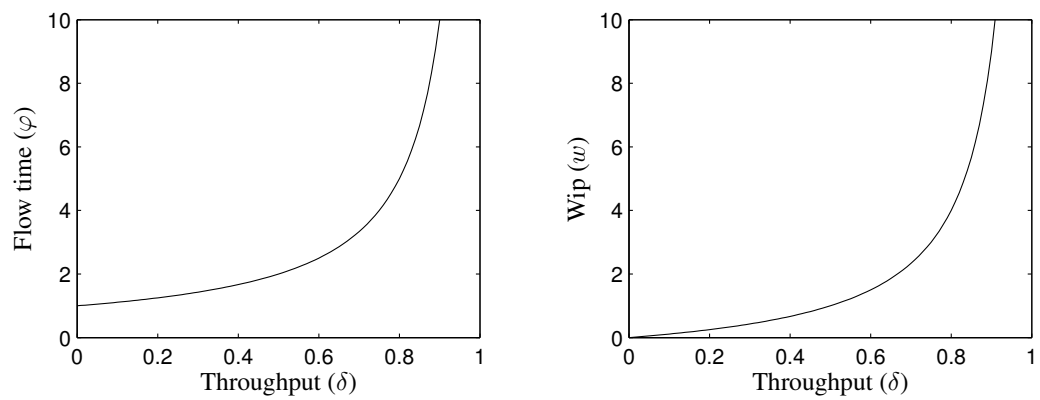

Figure 1.2: Basic relations between basic quantities for manufacturing systems

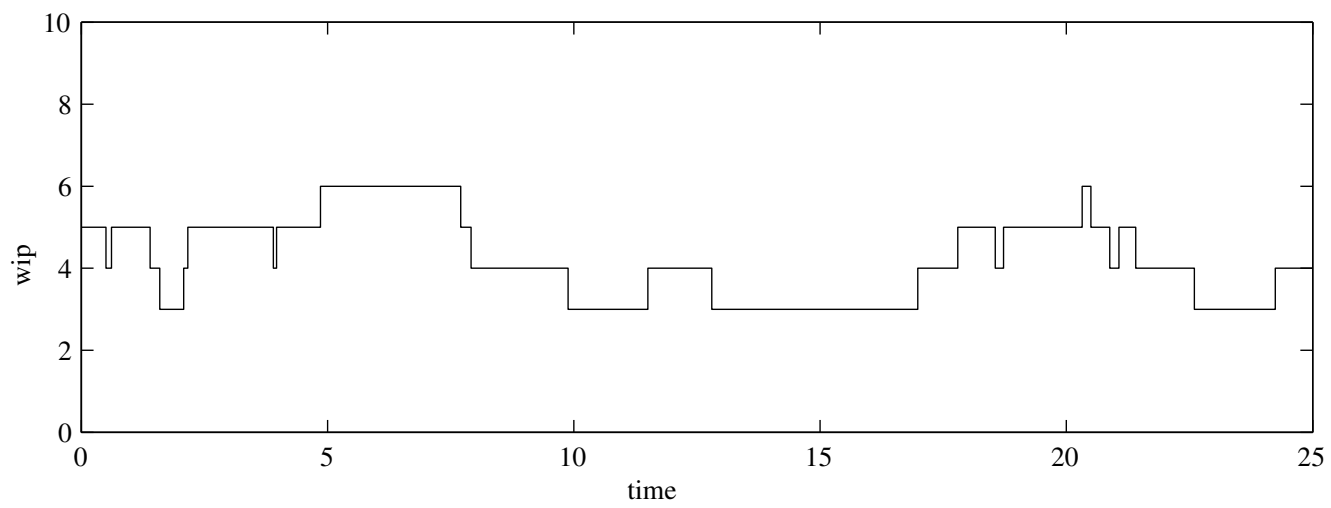

Figure 1.3: A characteristic time-behavior of wip at a workstation

When trying to control manufacturing systems, a trade-off needs to be made between throughput and flow time, so the nonlinear (steady state) relations depicted in Figure 1.2 need to be incorporated in any reasonable model of manufacturing systems.

A final observation of relevance for modeling manufacturing systems is the nature of the system signals. In Figure 1.3 a characteristic graph of the wip at a workstation as a function of time is shown. Wip always takes integer values with arbitrary (non-negative real) duration. One could consider a manufacturing system to be a system that takes values from a finite set of states and jumps from one state to the other as time evolves. This jump from one state to the other is called event. As we have a countable (discrete) number of states, it is clear that discrete event models are often used in modeling manufacturing systems. Discrete event models for manufacturing systems are considered in Section 1.4. But first some analytical models for analyzing steady state behavior of manufacturing systems are presented in the next section.

\subsection{Analytical models for steady state analysis}

In order to get some insights in the steady state performance of a given manufacturing system simple relations can be used. In this section we deal with mass conservation for determining the mean utilization of workstations and the number of machines required for meeting a required throughput. Furthermore, relations from queueing theory are used to obtain estimates for the mean wip and mean flow time. 


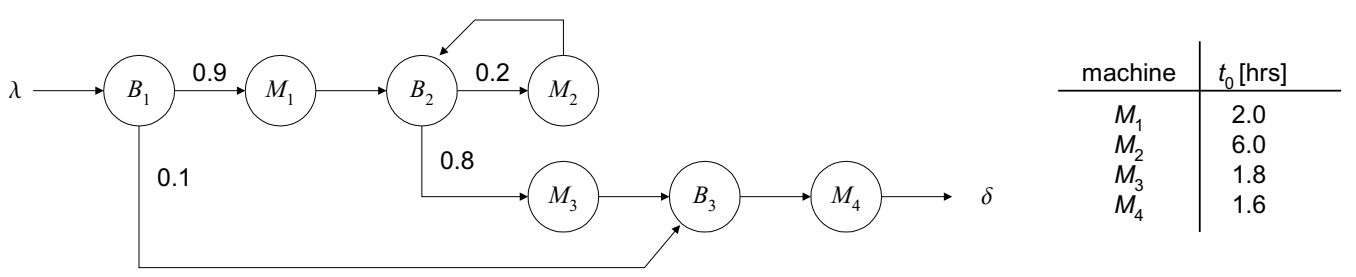

Figure 1.4: Manufacturing system with rework and bypassing

\subsubsection{Mass conservation (throughput)}

Using mass conservation the mean utilization of workstations can be easily determined. This is illustrated by means of an example.

Example 1.3.1. Consider the manufacturing system with rework and bypassing in Figure 1.4. The manufacturing system consists of three buffers and four machines. Lots are released at a rate of $\lambda$ lots/hour. The numbers near the arrows indicate the fraction of the lots that follow that route. For instance, of the lots leaving buffer $B_{1} 90 \%$ goes to machine $M_{1}$ and $10 \%$ goes to buffer $B_{3}$. The process time of each machine is listed in the table in Figure 1.4.

Let $\delta_{M_{i}}$ and $\delta_{B_{i}}$ denote the throughput of respectively machine $M_{i}(i=1,2,3,4)$ and buffer $B_{i}$ $(i=1,2,3)$. Using mass conservation we obtain

$$
\begin{aligned}
\delta_{M_{1}} & =0.9 \delta_{B_{1}} & \delta_{B_{1}} & =\lambda \\
\delta_{M_{2}} & =0.2 \delta_{B_{2}} & \delta_{B_{2}} & =\delta_{M_{1}}+\delta_{M_{2}} \\
\delta_{M_{3}} & =0.8 \delta_{B_{2}} & \delta_{B_{3}} & =\delta_{M_{3}}+0.1 \delta_{B_{1}} \\
\delta_{M_{4}} & =\delta_{B_{3}} & \delta & =\delta_{M_{4}} .
\end{aligned}
$$

Solving these linear relations results in:

$$
\begin{aligned}
\delta_{M_{1}} & =0.9 \lambda & \delta_{B_{1}} & =\lambda \\
\delta_{M_{2}} & =0.225 \lambda & \delta_{B_{2}} & =1.125 \lambda \\
\delta_{M_{3}} & =0.9 \lambda & \delta_{B_{3}} & =\lambda \\
\delta_{M_{4}} & =\lambda & \delta & \delta .
\end{aligned}
$$

Using the process times of the table in Figure 1.4, we obtain for the utilizations:

$$
\begin{aligned}
& u_{M_{1}}=0.9 \lambda \cdot 2.0 / 1=1.8 \lambda \\
& u_{M_{3}}=0.9 \lambda \cdot 1.8 / 1=1.62 \lambda \\
& u_{M_{2}}=0.225 \lambda \cdot 6.0 / 1=1.35 \lambda \\
& u_{M_{4}}=\lambda \cdot 1.6 / 1=1.6 \lambda .
\end{aligned}
$$

Clearly, machine $M_{1}$ is the bottleneck and the maximal throughput for this line is $\lambda=1 / 1.8=0.56$ jobs per hour.

Using mass conservation, utilizations of workstations can be determined straightforwardly. This also provides a way for determining the number of machines required for meeting a given throughput. By modifying the given percentages the effect of rework or a change in product mix can also be studied.

\subsubsection{Queueing relations (wip, flow time)}

Using mass conservation the mean utilization of a workstation can be determined. For determining a rough estimate of the corresponding mean flow time and mean wip, basic relations from queueing theory can be used.

Consider a single machine workstation that consists of infinite buffer $B_{\infty}$ and machine $M$, see Figure 1.5. Lots arrive at the buffer with a stochastic inter arrival time. The inter arrival time distribution has mean $t_{\mathrm{a}}$ and coefficient of variation $c_{\mathrm{a}}$. The coefficient of variation $c$ of a distribution is defined as the quotient of its standard deviation $\sigma$ and its mean $\mu: c=\sigma / \mu$. The machine has stochastic process times, with mean process time $t_{0}$ and coefficient of variation $c_{0}$. Finished lots leave the machine with a stochastic inter departure time, with mean $t_{\mathrm{d}}$ and 


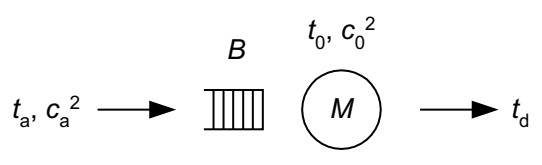

Figure 1.5: Single machine workstation

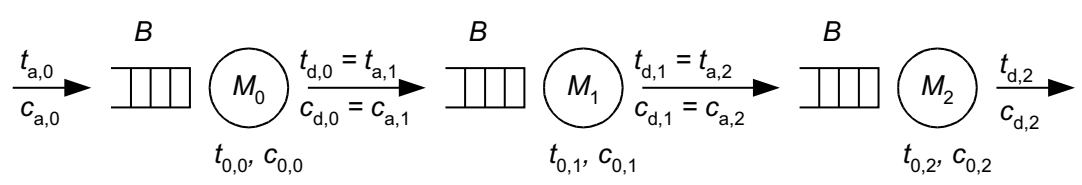

Figure 1.6: Three workstation flow line

coefficient of variation $c_{\mathrm{d}}$. Assuming independent inter arrival times and independent process times, the mean waiting time $\varphi_{B}$ in buffer $B$ can be approximated for a stable system by means of Kingman's equation (Kingman 1961):

$$
\varphi_{B}=\frac{c_{\mathrm{a}}^{2}+c_{0}^{2}}{2} \cdot \frac{u}{1-u} \cdot t_{0}
$$

with the utilization $u$ defined by:

$$
u=\frac{t_{0}}{t_{\mathrm{a}}}
$$

Equation 1.1 is exact for an $M / G / 1$ system, i.e. a single machine workstation with exponentially distributed inter arrival times and any distribution for the process time. For other single machine workstations it is an approximation.

For a stable system, we have $t_{\mathrm{d}}=t_{\mathrm{a}}$. We can approximate the coefficient of variation $c_{\mathrm{d}}$ by Kuehn's linking equation (Kuehn 1979):

$$
c_{\mathrm{d}}^{2}=\left(1-u^{2}\right) \cdot c_{\mathrm{a}}^{2}+u^{2} \cdot c_{0}^{2} .
$$

This result is exact for an $M / M / 1$ system. For other single machine workstations it is an approximation. Having characterized the departure process of a workstation, the arrival process at the next workstation has been characterized as well. As a result, a line of workstations can also be dealt with.

Example 1.3.2 (Three workstations in line). Consider the three workstation flow line from Figure 1.6. For the inter arrival time at workstation 0 we have $t_{\mathrm{a}}=4.0$ hours and $c_{\mathrm{a}}^{2}=1$. The three workstations are identical with respect to the process times: $t_{0, i}=3.0$ hours for $i=0,1,2$ and $c_{0, i}^{2}=0.5$ for $i=0,1,2$. Determine the mean total flow time per lot.

Since $t_{\mathrm{a}}>t_{0, i}$ for $i=0,1,2$, we have a stable system and $t_{a, i}=t_{d, i}=4.0$ hours for $i=0,1,2$. Subsequently, the utilization for each workstation is $u_{i}=3.0 / 4.0=0.75$ for $i=0,1,2$. We calculate the mean flow time for workstation 0, using Equation 1.1.

$$
\varphi_{0}=\varphi_{\mathrm{B}}+t_{0}=\frac{c_{\mathrm{a}}^{2}+c_{0}^{2}}{2} \cdot \frac{u}{1-u} \cdot t_{0}+t_{0}=\frac{1+0.5}{2} \cdot \frac{0.75}{1-0.75} \cdot 3.0+3.0=9.75 \text { hours }
$$

Next, we determine the coefficient of variation on the inter arrival time $c_{\mathrm{a}, 1}$ for workstation $W_{1}$ using Equation 1.2.

$$
c_{\mathrm{a}, 1}^{2}=c_{\mathrm{d}, 0}^{2}=\left(1-u^{2}\right) \cdot c_{\mathrm{a}}^{2}+u^{2} \cdot c_{0}^{2}=\left(1-0.75^{2}\right) \cdot 1+0.75^{2} \cdot 0.5=0.719 .
$$

We calculate the mean flow time for workstation 1 .

$$
\varphi_{1}=\frac{0.719+0.5}{2} \cdot \frac{0.75}{1-0.75} \cdot 3.0+3.0=8.49 \text { hours. }
$$


In a similar way, we determine that $c_{a, 2}^{2}=0.596, \varphi_{2}=7.93$ hours. We then calculate the mean total flow time.

$$
\varphi_{\text {tot }}=\varphi_{0}+\varphi_{1}+\varphi_{2}=26.2 \text { hours. }
$$

Note that the minimal flow time without variability $\left(c_{\mathrm{a}}^{2}=c_{0, i}^{2}=0\right)$ equals 9.0 hours.

Equations 1.1 and 1.2 are particular instances for a workstation consisting of a single machine. For workstations consisting of $m$ identical machines in parallel the following approximations can be used:

$$
\begin{aligned}
& \varphi_{B}=\frac{c_{\mathrm{a}}^{2}+c_{0}^{2}}{2} \cdot \frac{u^{\sqrt{2(m+1)}-1}}{m(1-u)} \cdot t_{0} \\
& c_{d}^{2}=\left(1-u^{2}\right) \cdot c_{\mathrm{a}}^{2}+u^{2} \cdot \frac{c_{0}^{2}+\sqrt{m}-1}{\sqrt{m}} .
\end{aligned}
$$

Notice that in case $m=1$ these equations reduce to Equation 1.1 and Equation 1.2.

Once the mean flow time has been determined, a third basic relation from queueing theory, Little's law (Little 1961), can be used for determining the mean wip level. Little's law states that the mean wip level (number of lots in a manufacturing system) $w$ is equal to the product of the mean throughput $\delta$ and the mean flow time $\varphi$, provided the system is in steady state.

$$
w=\delta \cdot \varphi \text {. }
$$

An example illustrates how Kingman's equation and Little's law can be used.

Example 1.3.3. Consider the system of Example 1.3.2 as depicted in Figure 1.6. From Example 1.3.2 we know that the flow times for the three workstations are respectively

$$
\varphi_{0}=9.75 \text { hours, } \quad \varphi_{1}=8.49 \text { hours, } \quad \varphi_{2}=7.93 \text { hours. }
$$

Since the steady state throughput was assumed to be $\delta=1 / t_{\mathrm{a}}=1 / 4.0=0.25$ lots/hour, we obtain via Little's law

$$
\begin{aligned}
& w_{0}=0.25 \cdot 9.75=2.44 \text { lots } \\
& w_{1}=0.25 \cdot 8.49=2.12 \text { lots } \\
& w_{2}=0.25 \cdot 7.93=1.98 \text { lots }
\end{aligned}
$$

The above mentioned relations are simple approximations that can be used for getting a rough idea about the possible performance of a manufacturing system. These approximations are fairly accurate for high degrees of utilization but less accurate for lower degrees of utilization. A basic assumption when using these approximations is independence of the inter arrival times, which in general is not the case, e.g. for merging streams of jobs. Furthermore, using these equations only steady state behavior can be analyzed. For studying things like ramp-up behavior or for incorporating more details like operator-behavior, more sophisticated models are needed, as described in the next section.

\subsection{Discrete event models}

In the previous section simple methods have been introduced for analyzing steady state behavior of manufacturing systems. For analyzing the dynamics of manufacturing systems, more sophisticated models are required. In Figure 1.3 in Section 1.2 it was illustrated that typical models of manufacturing systems are so-called discrete event models. In this section we illustrate by means of an example how to build a discrete event model of a manufacturing system using the specification language $\chi$, which is explained in more detail in Chapter XXX of this Handbook.

The way to build a discrete event model is to consider the manufacturing system as a network of concurrent processes through which jobs and other types of information flows. For example, a 
basic machine can be modeled as a process which repeatedly tries to receive a lot, delays for the period of time (the process time), and tries to send a lot. Using $\chi$, we can write

$$
\operatorname{proc} M\left(\operatorname{chan} a ?, b !: \text { lot, } \operatorname{disc} t_{e}: \text { real }\right)=\|\left[\operatorname{disc} x: \operatorname{lot}:: *\left(a ? x ; \Delta t_{e} ; b ! x\right)\right] \mid \text {. }
$$

The machine is able to receive lots via external channel $a$, is able to send lots via external channel $b$ and the process time of the machine is given by parameter $t_{e}$. Repeatedly the machine tries to receive a lot over external channel $a$ and store this lot in discrete variable $x$. Next, the machine delays for $t_{e}$, after which the machine tries to send $x$ via external channel $b$.

A buffer can be modeled as a process that as long as it can store something it is willing to receive new lots and as long as it has something stored is willing to send lots. Using $\chi$ a finite FIFO (first in, first out) buffer with buffer size $n$ can be modeled as

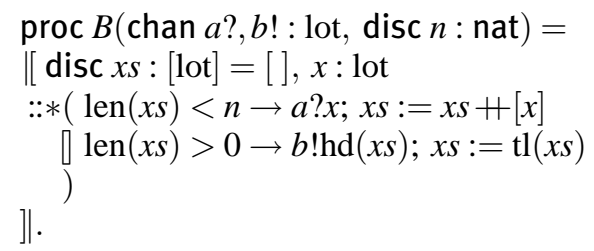

This process can receive lots via external channel $a$, send lots via external channel $b$ and has its maximal buffer size $n$ as a parameter. Repeatedly two alternatives can be executed.

- On the one hand receiving a lot via channel $a$ (only if the length of the list is less than $n$ ) into discrete variable $x$ and consecutively adding it in the proper way to list $x s$ of lots (using a concatenation of lists).

- On the other hand trying to send the head of the list (its first element) via channel $b$ (only if the list is not empty) and consecutively reducing list $x s$ to its tail (everything but the first element).

Next, these two processes can be used to model a workstation which consists of a 3-place buffer and a machine with process time $t_{e}$ by simply putting the two previously specified processes in parallel:

$$
\operatorname{proc} W\left(\operatorname{chan} a ?, b !: \text { lot, disc } t_{e}: \text { real }\right)=\|\left[\operatorname{chan} c: \operatorname{lot}:: B(a, c, 3) \| M\left(c, b, t_{e}\right)\right] \mid .
$$

Assume that lots arrive to this workstation according to a Poisson arrival process with mean arrival rate of $\lambda$ jobs per unit time. This can be modeled by means of the following generator process

$$
\begin{aligned}
& \operatorname{proc} G(\text { chan } a !: \text { lot, disc } \lambda: \text { real })= \\
& \|[\operatorname{disc} u: \rightarrow \text { real }=\operatorname{exponential}(1 / \lambda):: *(a ! \tau ; \Delta \sigma u ; b ! x)] \|
\end{aligned}
$$

where we assumed that the type lot consists of a real number which contains the time this lot entered the system. Generator $G$ is able to send lots via external channel $a$ and has a mean departure rate which is given by parameter $\lambda$. The discrete variable $u$ contains an exponential distribution with mean $1 / \lambda$. Repeatedly the generator tries to send a lot over external channel $a$, where the lot contains upon departure the current time $\tau$. Next, the generator delays for a period which is given by a sample from the distribution $u$.

To complete this specification, assume that lots leave to exit process $E$ after being served at workstation $W$. This exit process can be modelled as

$$
\operatorname{proc} E(\operatorname{chan} a ?: \operatorname{lot})=\|[\operatorname{disc} x: \operatorname{lot}:: *(a ? x)] \mid \text {. }
$$

This process repeatedly tries to receive a lot via external channel $a$.

If we assume an arrival rate of $\lambda=0.5$ and a process time of $t_{e}=1.5$ the specification of the discrete event model can be completed by specifying

$$
\text { model } G W E()=\| \text { chan } a, b: \operatorname{lot}:: G(a, 0.5)\|W(a, b, 1.5)\| E(b)] \mid .
$$

In this way a manufacturing system can be modeled as a network of concurrent processes through which jobs and other types of information flows. The presented model is rather simple, 


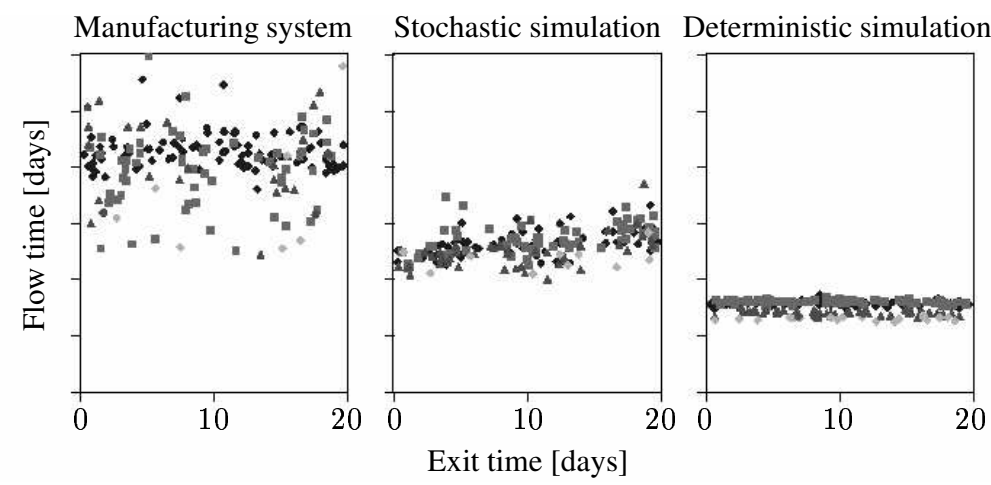

Figure 1.7: A comparison

but clearly many more ingredients can be added. For example, an operator might be required during processing of a machine. To include this, we can modify the proces $M$ into

$$
\begin{aligned}
& \operatorname{proc} \bar{M}\left(\text { chan } a \text { ? }, b !: \text { lot, } c \text { ?, } d !: \text { operator, disc } t_{e}: \text { real }\right)= \\
& \|\left[\operatorname{disc} x: \text { lot, } y: \text { operator }:: *\left(c ? y ; a ? x ; \Delta t_{e} ; b ! x ; d ! y\right)\right] \mid
\end{aligned}
$$

Highly detailed models of manufacturing systems can be made in this way, even before the system has been build. The influence of certain parameters can be analyzed by running several experiments with the discrete event model using different parameter settings. This is common practice when designing a several billion wafer fab for instance. Since in practice manufacturing systems are changing continuously, it is very hard to keep these detailed discrete event models up-to-date.

Fortunately, for a manufacturing system in operation it is possible to arrive at more simple/less detailed discrete event models by using the concept of effective process times (EPT's) as introduced in the next section.

\subsection{Effective process times (EPT's)}

As mentioned in the previous section, for the processing of a lot at a machine, many steps may be required. It could be that an operator needs to get the lot from a storage device, setup a specific tool that is required for processing the lot, put the lot on an available machine, start a specific program for processing the lot, wait until this processing has finished (meanwhile doing something else), inspect the lot to determine if all went well, possibly perform some additional processing (e.g. rework), remove the lot from the machine and put it on another storage device and transport it to the next machine. At all of these steps something might go wrong: the operator might not be available, after setting up the machine the operator finds out that the required recipe can not be run on this machine, the machine might fail during processing, no storage device is available anymore so the machine can not be unloaded and is blocked, etc. It is impossible to measure all sources of variability that might occur in a manufacturing system. One could incorporate some of these sources in a discrete event model. The number of operators and tools can be modeled explicitly and it is common practice to collect data on mean times to failure and mean times to repair of machines. Also schedules for (preventive) maintenance can be incorporated explicitly in a discrete event model. Nevertheless, still not all sources of variability are included. This is clearly illustrated in Figure 1.7, obtained from (Jacobs, Etman, Campen, and Rooda 2003). The left graph contains actual realizations of flow times of lots leaving a real manufacturing system, whereas the right graph contains the results of a detailed deterministic simulation model and the graph in the middle contains the results of a similar model including stochasticity. It turns out that in reality flow times are much higher and much more irregular than simulation predicts. So, even if one tries hard to capture all variability 


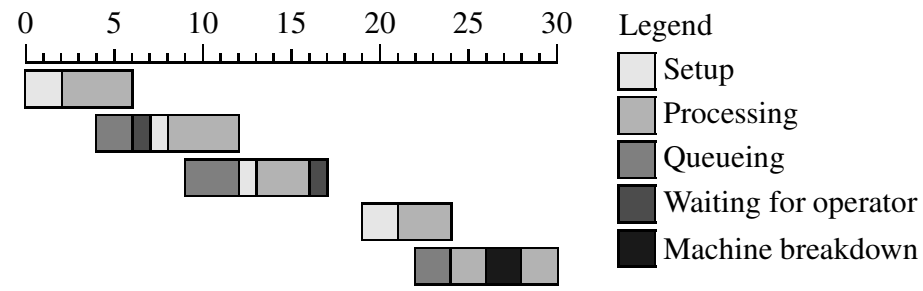

Figure 1.8: Gantt chart of 5 lots at a single machine workstation

present in a manufacturing system, still the outcome predicted by the model is far from reality. In (Hopp and Spearman 2000) the term effective process time (EPT) has been introduced as the time seen by lots from a logistical point of view. In order to determine this effective process time, Hopp and Spearman assume that the contribution of the individual sources of variability is known.

A similar description is given in (Sattler 1996) where the effective process time has been defined as all flow time except waiting for another lot. It includes waiting for machine down time and operator availability and a variety of other activities. In (Sattler 1996) it was also noticed that this definition of effective process time is difficult to measure.

Instead of taking the bottom-up view of Hopp and Spearman, a top-down approach can also be taken, as shown by Jacobs et al. (Jacobs, Etman, Campen, and Rooda 2003), where algorithms have been introduced that enable determination of effective process time realizations from a list of events. For these algorithms, the basic idea of the effective process time to include time losses was used as a starting point.

Consider a single machine workstation (cf. Figure 1.5) and assume that the Gantt chart of Figure 1.8 describes what has happened at this workstation during a given period. At $t=0$ the first lot arrives at the workstation. After a setup, the processing of the lot starts at $t=2$ and is completed at $t=6$. At $t=4$ the second lot arrives at the workstation. At $t=6$ this lot could have been started, but apparently there was no operator available, so only at $t=7$ the setup for this lot starts. Eventually, at $t=8$ the processing of the lot starts and is completed at $t=12$. The fifth lot arrives at the workstation at $t=22$, processing starts at $t=24$, but at $t=26$ the machine breaks down. It takes until $t=28$ before the machine has been repaired and the processing of the fifth lot continues. The processing of the fifth lot is completed at $t=30$.

If we take the point of view of a lot, what does a lot see from a logistical point of view? The first lot arrives at an empty system at $t=0$ and departs from this system at $t=6$. From the point of view of this lot, its processing took 6 time-units. The second lot arrives at a non-empty system at $t=4$. Clearly, this lot needs to wait. However, at $t=6$, if we would forget about the second lot, the system becomes empty again. So from $t=6$ on there is no need for the second lot to wait. At $t=12$ the second lot leaves the system, so from the point of view of this lot, its processing took from $t=6$ till $t=12$; the lot does not know whether waiting for an operator and a setup is part of its processing. Similarly, the third lot sees no need for waiting after $t=12$ and leaves the system at $t=17$, so it assumes to have been processed from $t=12$ till $t=17$. Following this reasoning, the resulting effective process times for lots are as depicted in Figure 1.9. Notice that only arrival and departure events of lots to a workstation are needed for determining the effective process times. Furthermore, none of the contributing disturbances needs to be measured. In highly automated manufacturing systems, arrival and departure events of lots are being registered, so for these manufacturing systems, effective process time realizations can be determined rather easily. Next, these EPT realizations can be used in a relatively simple discrete event model of the manufacturing system. This discrete event model only contains the architecture of the manufacturing system, buffers and machines. The process times of these machines are samples from their EPT-distribution as measured from real manufacturing data. There is no need for incorporating machine failures, operators, etc., as this is all included in the EPT-distributions. Furthermore, the algorithms as provided in (Jacobs, Etman, Campen, and 


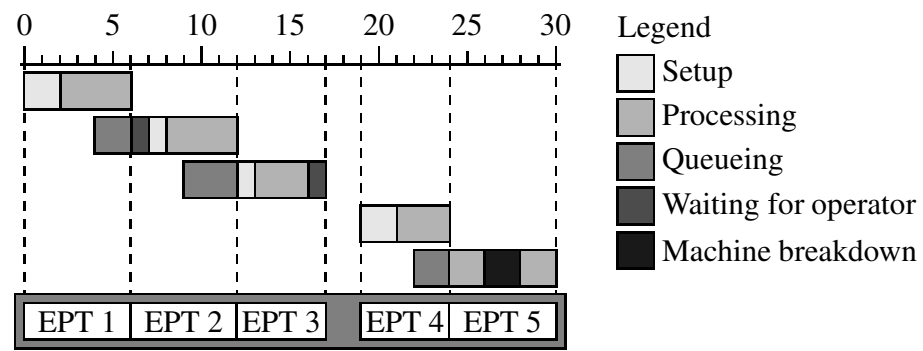

Figure 1.9: EPT realizations of 5 lots at a workstation

Rooda 2003) are utilization independent. That is, data collected at a certain throughput rate is also valid for different throughput rates. Also, machines with the same EPT-distribution can be added to a workstation. This makes it possible to study how the manufacturing system responds in case a new machine is added, or all kinds of other what-if-scenario's. Finally, since EPT-realizations characterize operational time variability, they can be used for performance measuring. For more on this issue, the interested reader is referred to (Jacobs, Etman, Campen, and Rooda 2003; Jacobs, Bakel, Etman, and Rooda 2005). What is most important is that EPT's can be determined from real manufacturing data and yield relatively simple discrete event models of the manufacturing system under consideration. These relatively simple discrete event models can serve as a starting point for controlling manufacturing systems dynamically.

\subsection{Control of manufacturing systems: a framework}

In the previous section, the concept of effective process times has been introduced as a means to arrive at relatively simple discrete event models for manufacturing systems, using measurements from the real manufacturing system under consideration. The resulting discrete event models are large queueing networks which capture the dynamics reasonably well. These relatively simple discrete event models are not only a starting point for analyzing the dynamics of a manufacturing system, but can also be used as a starting point for controller design. If one is able to control the dynamics of the discrete event model of the manufacturing system, the resulting controller can also be used for controlling the real manufacturing system.

Even though control theory exists for controlling discrete event systems, unfortunately none of it is appropriate for controlling discrete event models of real-life manufacturing systems. This is mainly due to the large number of states a manufacturing system can be in. Therefore, a different approach is needed.

If we concentrate on mass production, the distinction between lots is not really necessary and lots can be viewed in a more continuous way. Therefore, instead of the discrete event model we consider an approximation model. Some of these approximation models are presented in the next two sections.

Once we have an approximation model, we can use standard control theory for deriving a controller for the approximation model, cf. Figure 1.10.

When the derived controller behaves as desired, i.e. when the closed-loop system of approximation model and controller behaves as desired, the derived controller can be connected to the discrete event model. This can not be done straightforwardly, since the derived controller is not a discrete event controller. The control actions still need to be transformed into events. It might very well be that the optimal control action would be to produce 2.75 lots during the next shift. One still needs to decide how many jobs to really start (2 or 3), and also when to start them. This is the left conversion block in Figure 1.11. From this figure, it can also be seen that a conversion is needed from discrete event model to controller. A simple conversion would be to sample the discrete event model once every shift. But also other strategies might be followed. For example, if at the beginning of a shift a machine breaks down it might not be such a good 


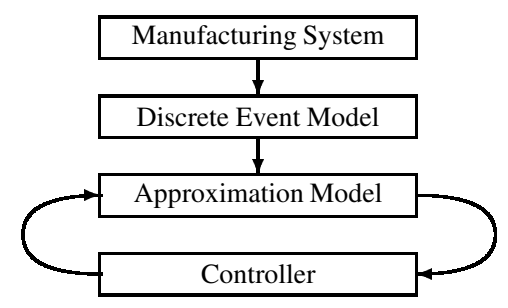

Figure 1.10: Control framework (I)

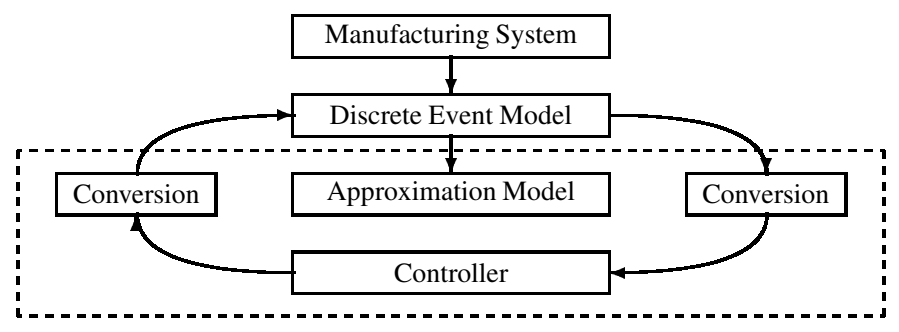

Figure 1.11: Control framework (II)

idea to wait until the end of the shift before setting new production targets. Therefore, the next step would be to designing proper conversion blocks.

Once the two conversion blocks have been properly designed a suitable discrete event controller for the discrete event model is obtained, as illustrated in Figure 1.11 (dashed).

Eventually, as a final step, the designed controller can be disconnected from the discrete event model, and attached to the real manufacturing system.

In the presented control framework two crucial steps can be distinguished. First, the discrete event model should be a good enough approximation of the real manufacturing system. For that reason, once a discrete event model of a manufacturing system has been made, the model needs to be validated. If results as shown in Figure 1.7 are obtained the model needs further improvement. Second, the approximation model should be a good enough approximation of the discrete event model, or actually: of the discrete event model and conversion block(s), since that is the system that needs to be controlled by the continuous controller. Depending on the variables of interest, a valid approximation model needs to be used. An overview of common used approximation models, assuming mass production, is provided in the next two sections. In section 1.7 approximation models are presented that mainly focus on throughput. In section 1.8 approximation models are presented that incorporate both throughput and flow time, taking into account the nonlinear relations as depicted in Figure 1.2.

\subsection{Standard fluid model and extensions}

A disadvantage of the analytical approximations models as presented in Section 1.3 is that they are only concerned with steady state. No dynamics is included. This disadvantage is overcome by discrete event models as introduced in Section 1.4, but the price to be paid is the long simulation times that are required for obtaining satisfactory results. This is due to the fact that each job is modeled separately. As explained in the previous section we can overcome these long simulation times by using an approximation model. In this section some of the possible approximation models are presented. The main idea is to view the amount of jobs in a continuous way. This results in so called fluid models. 


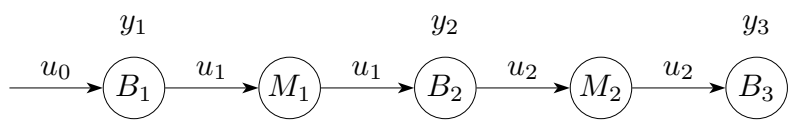

Figure 1.12: A simple manufacturing system

\subsubsection{A common fluid model}

The current standard way of deriving fluid models is most easily explained by means of an example. Therefore, consider a simple manufacturing system consisting of two machines in series, as displayed in Figure 1.12. Let $u_{0}(t)$ denote the rate at which jobs arrive to the system at time $t$, let $u_{i}(t)$ denote the rate at which machine $M_{i}$ produces lots at time $t$, let $y_{i}(t)$ denote the number of lots in buffer $B_{i}$ at time $t(i \in\{1,2\})$ and let $y_{3}(t)$ denote the number of lots produced by the manufacturing system at time $t$. Assume that machines $M_{1}$ and $M_{2}$ have a maximum capacity of respectively $\mu_{1}$ and $\mu_{2}$ lots per time unit. This provides us with all information for deriving a fluid model.

Clearly the rate of change of the buffer contents is given by the difference between the rates at which lots enter and leave the buffer. Under the assumption that the number of lots can be considered continuous, this observation leads to the following fluid model:

$$
\begin{aligned}
& \dot{y}_{1}(t)=u_{0}(t)-u_{1}(t), \\
& \dot{y}_{2}(t)=u_{1}(t)-u_{2}(t), \\
& \dot{y}_{3}(t)=u_{2}(t),
\end{aligned}
$$

which can also be expressed as follows:

$$
\begin{aligned}
& \dot{x}(t)=\left[\begin{array}{lll}
0 & 0 & 0 \\
0 & 0 & 0 \\
0 & 0 & 0
\end{array}\right] x(t)+\left[\begin{array}{ccc}
1 & -1 & 0 \\
0 & 1 & -1 \\
0 & 0 & 1
\end{array}\right] u(t), \\
& y(t)=\left[\begin{array}{lll}
1 & 0 & 0 \\
0 & 1 & 0 \\
0 & 0 & 1
\end{array}\right] x(t)+\left[\begin{array}{lll}
0 & 0 & 0 \\
0 & 0 & 0 \\
0 & 0 & 0
\end{array}\right] u(t),
\end{aligned}
$$

where $u=\left[u_{0}, u_{1}, u_{2}\right]^{\top}$ and $y=\left[y_{1}, y_{2}, y_{3}\right]^{\top}$. We also have capacity constraints on the input, as well as the constraint that the buffer contents should remain positive. These constraints can be expressed by means of the following equations:

$$
0 \leq u_{1}(t) \leq \mu_{1}, 0 \leq u_{2}(t) \leq \mu_{2} \quad \text { and } \quad y_{1}(t) \geq 0, y_{2}(t) \geq 0, y_{3}(t) \geq 0 .
$$

The system as described by Equation 1.7 is a controllable linear system of the form $\dot{x}=A x+B u, y=C x+D u$ as extensively studied in control theory.

Note that instead of a description in continuous time, a description in discrete time can also be used:

$$
\begin{aligned}
\dot{x}(k+1) & =\left[\begin{array}{lll}
1 & 0 & 0 \\
0 & 1 & 0 \\
0 & 0 & 1
\end{array}\right] x(k)+\left[\begin{array}{ccc}
1 & -1 & 0 \\
0 & 1 & -1 \\
0 & 0 & 1
\end{array}\right] u(k), \\
y(k) & =\left[\begin{array}{lll}
1 & 0 & 0 \\
0 & 1 & 0 \\
0 & 0 & 1
\end{array}\right] x(k)+\left[\begin{array}{ccc}
0 & 0 & 0 \\
0 & 0 & 0 \\
0 & 0 & 0
\end{array}\right] u(k) .
\end{aligned}
$$

Also, the description of Equation 1.7 is not the only possible input/output/state model which yields the input/output behavior Equation 1.6. To illustrate this, consider the change of coordinates

$$
x(t)=\left[\begin{array}{ccc}
1 & -1 & 0 \\
0 & 1 & -1 \\
0 & 0 & 1
\end{array}\right] \bar{x}(t),
$$




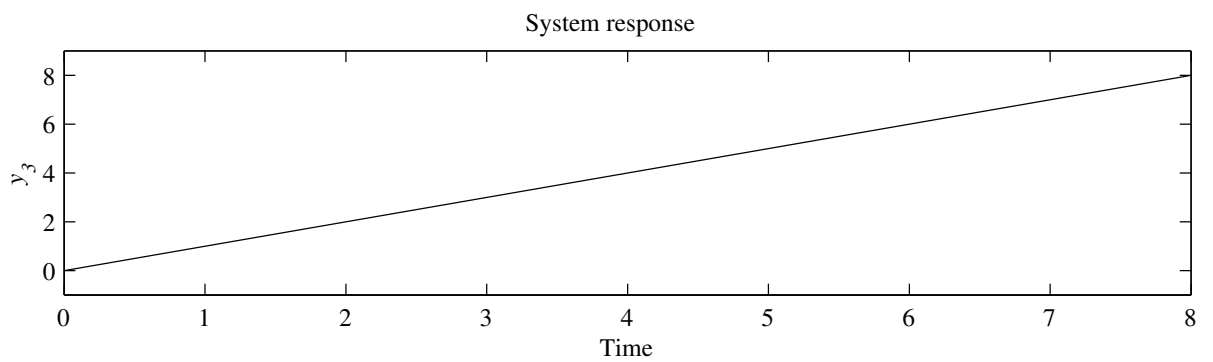

Figure 1.13: Output of the manufacturing system using model Equation 1.6

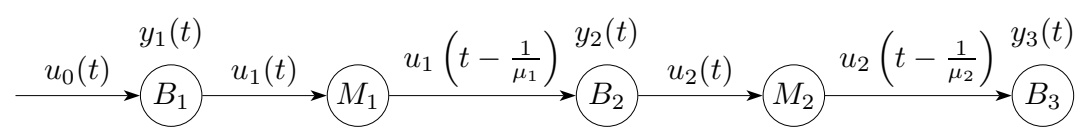

Figure 1.14: A simple manufacturing system revisited

which results in the following input/output/state model:

$$
\begin{aligned}
& \dot{\bar{x}}(t)=\left[\begin{array}{lll}
0 & 0 & 0 \\
0 & 0 & 0 \\
0 & 0 & 0
\end{array}\right] \bar{x}(t)+\left[\begin{array}{lll}
1 & 0 & 0 \\
0 & 1 & 0 \\
0 & 0 & 1
\end{array}\right] u(t), \\
& y(t)=\left[\begin{array}{ccc}
1 & -1 & 0 \\
0 & 1 & -1 \\
0 & 0 & 1
\end{array}\right] \bar{x}(t)+\left[\begin{array}{lll}
0 & 0 & 0 \\
0 & 0 & 0 \\
0 & 0 & 0
\end{array}\right] u(t) .
\end{aligned}
$$

Note that in this description, the state $\bar{x}$ denotes the cumulative production at each workstation. We would like to study the response of the output of the system Equation 1.7, or equivalently Equation 1.11. Assume that initially we start with an empty production line (i.e. $x(0)=0)$, that both machines have a capacity of 1 lot per unit time (i.e. $\mu_{1}=\mu_{2}=1$ ) and that we feed the line at a rate of 1 lot per time unit (i.e. $u_{0}=1$ ). Furthermore, assume that machines produce at full capacity, but only in case something is in the buffer in front of it, i.e.

$$
u_{i}(t)=\left\{\begin{array}{ll}
\mu_{i} & \text { if } y_{i}(t)>0 \\
0 & \text { otherwise }
\end{array} \quad i \in\{1,2\} .\right.
$$

Under these assumptions, the resulting contents of buffer $B_{3}$ are as displayed in Figure 1.13. Notice that immediately lots start coming out of the system. Clearly this is not what happens in practice. Since both machines $M_{1}$ and $M_{2}$ need to process the first lot, it should take the system at least $\frac{1}{\mu_{1}}+\frac{1}{\mu_{2}}$ time units before lots can come out. This illustrates that for the fluid model as given by Equation 1.7 or Equation 1.11 does not incorporate flow times.

\subsubsection{An extended fluid model}

In the previous subsection we noticed that in the standard fluid model lots immediately come out of the system, once we start producing. A way to overcome this problem is to explicitly take into account the required delay. Whenever we decide to change the production rate of machine $M_{1}$, buffer $B_{2}$ notices this $1 / \mu_{1}$ time units later. As a result the rate at which lots arrive to buffer $B_{2}$ at time $t$ is equal to the rate at which machine $M_{1}$ was processing at time $t-1 / \mu_{1}$. This observation results in the following model (see also Figure 1.14): 


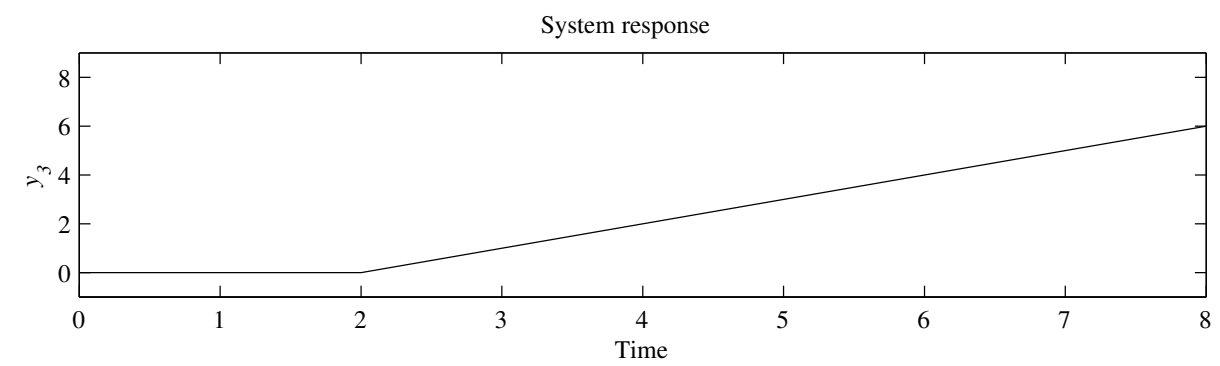

Figure 1.15: Output of the manufacturing system using model Equation 1.13

$$
\begin{aligned}
& \dot{y}_{1}(t)=u_{0}(t)-u_{1}(t), \\
& \dot{y}_{2}(t)=u_{1}\left(t-\frac{1}{\mu_{1}}\right)-u_{2}(t), \\
& \dot{y}_{3}(t)=u_{2}\left(t-\frac{1}{\mu_{2}}\right) .
\end{aligned}
$$

Clearly the constraints of Equation 1.8 also apply to the model given by Equation 1.13.

We expect that this model shows a response which is closer to reality. Assume that for the system described by Equation 1.13 we also have $\mu_{1}=\mu_{2}=1$ lot per time unit, and that we perform the same experiments as in the previous subsection, i.e. start from $x(0)=0$, apply $u_{0}=1$ and Equation 1.12. The resulting response of buffer $B_{3}$ is displayed in Figure 1.15. If we compare the results from Figure 1.15 to that of Figure 1.13 we see that no products enter buffer $B_{3}$ during the first 2.0 time units in case we use the extended fluid model. Clearly the extended fluid model produces more realistic results than the standard fluid model.

\subsubsection{An approximation to the extended fluid model}

In the previous subsection we proposed an extended version of the standard fluid model. Although the model of Equation 1.13 still is a linear model, standard linear control theory is not able to deal with this model, due to the time delay. For controlling the model of Equation 1.13 we have to rely on control theory of infinite-dimensional linear systems. For a good introduction to infinite-dimensional linear systems, see e.g. (Curtain and Zwart 1995).

Instead of using infinite-dimensional linear systems theory, another possibility would be to approximate the time delays by means of a Padé approximation (Baker 1965). When we use second-order Padé approximations, the model of Equation 1.13 can be approximated as:

$$
\begin{aligned}
\dot{x} & =\left[\begin{array}{ccccccc}
\mathbf{0} & \mathbf{0} & 0 & 0 & \mathbf{0} & 0 & 0 \\
\mathbf{0} & \mathbf{0} & 0 & 0 & \mathbf{0} & 0 & 0 \\
0 & 0 & 4 & 6 & -3 & 0 & 0 \\
0 & 0 & 0 & 4 & 0 & 0 & 0 \\
\mathbf{0} & \mathbf{0} & 0 & 0 & \mathbf{0} & 0 & 0 \\
0 & 0 & 0 & 0 & 4 & 6 & -3 \\
0 & 0 & 0 & 0 & 0 & 4 & 0
\end{array}\right] x+\left[\begin{array}{ccc}
\mathbf{1} & \mathbf{0} & \mathbf{0} \\
\mathbf{0} & \mathbf{1} & \mathbf{0} \\
0 & 0 & 0 \\
0 & 0 & 0 \\
\mathbf{0} & \mathbf{0} & \mathbf{1} \\
0 & 0 & 0 \\
0 & 0 & 0
\end{array}\right] u, \\
y & =\left[\begin{array}{ccccccc}
\mathbf{1} & -\mathbf{1} & 0 & 0 & \mathbf{0} & 0 & 0 \\
\mathbf{0} & \mathbf{1} & -3 & 0 & -\mathbf{1} & 0 & 0 \\
\mathbf{0} & \mathbf{0} & 0 & 0 & \mathbf{1} & -3 & 0
\end{array}\right] x+\left[\begin{array}{ccc}
\mathbf{0} & \mathbf{0} & \mathbf{0} \\
\mathbf{0} & \mathbf{0} & \mathbf{0} \\
\mathbf{0} & \mathbf{0} & \mathbf{0}
\end{array}\right] u .
\end{aligned}
$$

Notice the structure in Equation 1.14. In bold face we can easily recognize the dynamics of Equation 1.11. The additional dynamics results from approximating the time delays by means of a second order Padé approximation.

If we initiate the system of Equation 1.14 from $x(0)=0$ and feed it at a rate $u_{0}=1$ while using Equation 1.12, we obtain the system response as depicted in Figure 1.16. It is clear that we do not get the same response as in Figure 1.15, but the result is rather acceptable from a practical 


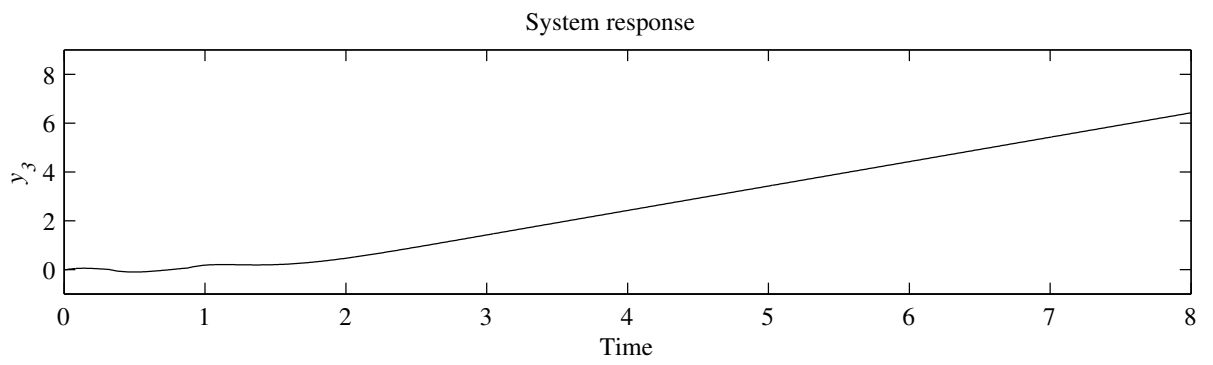

Figure 1.16: Output of the manufacturing system using model Equation 1.14

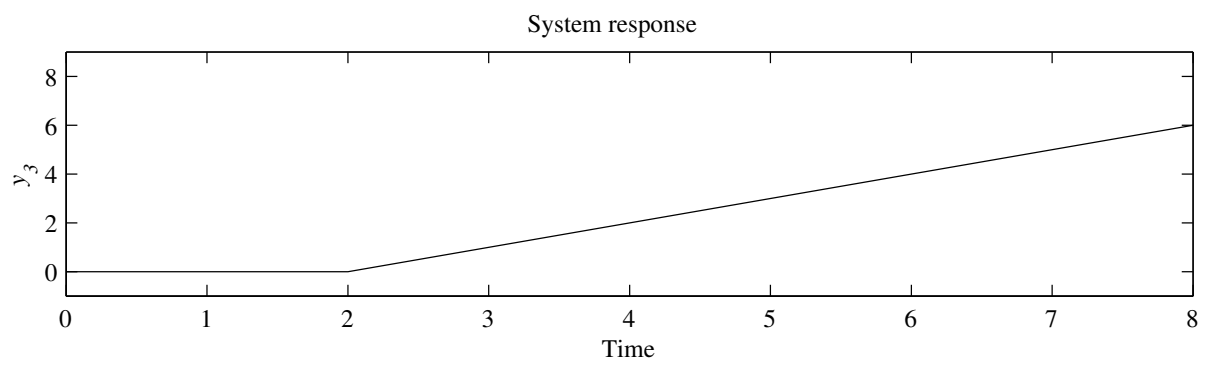

Figure 1.17: Output of the manufacturing system

point of view. At least it is closer to reality than the response as displayed in Figure 1.13.

\subsubsection{A hybrid model}

In the previous subsections, we provided some extensions to the standard fluid model by taking into account the time delay lots encounter due to the processing of machines. We also mentioned the constraints of Equation 1.8 that have to be obeyed. These are constraints that we have to take into account when designing a controller for our manufacturing system. The way we dealt with these constraints in the previous subsections was by requiring the machines to produce only in case the buffer contents in front of that machine were positive, cf. Equation 1.12.

A way to extend the standard fluid model Equation 1.7 is to think of these constraints in a different way. As illustrated in subsection 1.7.1, when we turn on both machines, immediately lots start coming out of the system. This is an undesirable feature that we would like to avoid. In practice, the second machine can only start producing when the first machine has finished a lot. Keeping this in mind, why do we allow machine $M_{2}$ to start producing as soon as the buffer contents of the buffer in front of it are positive? Actually, machine $M_{2}$ should only start producing as soon as a whole product has been finished by the machine $M_{1}$. In words: machine $M_{2}$ should only start producing as soon as the buffer contents of the buffer in front of it becomes 1. Therefore, we should not allow for a positive $u_{2}$ as soon as $y_{2}>0$, but only in case $y_{2} \geq 1$. When we consider the initially empty system Equation 1.7, i.e. $x(0)=0$, and assume

$$
u_{i}(t)=\left\{\begin{array}{ll}
\mu_{i} & \text { if } y_{i}(t) \geq 1 \\
0 & \text { otherwise }
\end{array} \quad i \in\{1,2\}\right.
$$

the resulting system response to an input of $u_{0}=1$ is shown in Figure 1.17. Notice that we obtain exactly the same response as in Figure 1.15.

Unfortunately, this is not all. The change in the constraints as proposed is not sufficient. It is in case we ramp up our manufacturing systems, but in case we ramp down it is not. Suppose that after a while we do not feed the manufacturing line any more, i.e. after a while we have $u_{0}=0$. In that case machine $M_{1}$ builds off the contents of the buffer $B_{1}$, until exactly one product 
remains. As soon as $y_{1}=1$, the machine is not allowed to produce any more due to the constraint we imposed. This is not what we would like to have. Therefore, in case $u_{1}=0$, machine $M_{1}$ should be allowed to produce until $y_{1}=0$.

Under these conditions, we could also think of our model operating in different modes. For the manufacturing system under consideration we can distinguish the following modes:

$$
\begin{aligned}
& \text { mode 1: } \quad 0 \leq y_{1} \leq 1, \quad 0 \leq y_{2} \leq 1, \quad u_{0}=0, \quad u_{1} \geq 0, \quad u_{2}=0 . \\
& \text { mode 2: } \quad 0 \leq y_{1} \leq 1, \quad 0 \leq y_{2} \leq 1, \quad u_{0} \geq 0, \quad u_{1}=0, \quad u_{2} \geq 0 \text {. } \\
& \text { mode 3: } \quad 1 \leq y_{1}, \quad 0 \leq y_{2} \leq 1, \quad u_{1}=0, \quad u_{2} \geq 0 \text {. } \\
& \text { mode 4: } \quad 1 \leq y_{1}, \quad 0 \leq y_{2} \leq 1, \quad u_{1} \geq 0, \quad u_{2}=0 \text {. } \\
& \text { mode 5: } 0 \leq y_{1} \leq 1, \quad 1 \leq y_{2}, \quad u_{0}=0, \quad u_{1} \geq 0 \text {. } \\
& \text { mode 6: } 0 \leq y_{1} \leq 1, \quad 1 \leq y_{2}, \quad u_{0} \geq 0, \quad u_{1}=0 \text {. } \\
& \text { mode 7: } \quad 1 \leq y_{1}, \quad 1 \leq y_{2}
\end{aligned}
$$

In all of these modes, the system dynamics is described by Equation 1.7.

In fact, what we just presented is a hybrid systems model of the manufacturing system under consideration. The description as just presented is also known as that of piecewise affine (PWA) systems (Sontag 1981). Other well-known descriptions are linear complementarity (LC) systems (Heemels, Schumacher, and Weiland 2000; Schaft and Schumacher 1998) and mixed logical dynamical (MLD) systems (Bemporad and Morari 1999). In (Bemporad, Ferrari-Trecate, and Morari 2000; Heemels, Schutter, and Bemporad 2001) it was shown that (under certain assumptions like well-posedness) these three descriptions are equivalent. This knowledge is useful, as each modeling class has its own advantages. Stability criteria for PWA systems were proposed in (DeCarlo, Branicky, Petterson, and Lennartson 2000; Johansson and Rantzer 1998), and control and state-estimation techniques for MLD hybrid models have been presented in (Bemporad, Borrelli, and Morari 2000; Bemporad, Mignone, and Morari 1999; Bemporad and Morari 1999). These results can now be applied for controlling the hybrid systems model of our manufacturing system.

\subsection{Flow models}

In the previous section we introduced the fluid model described by Equation 1.6. A shortcoming of this model was that once we start producing, immediately lots come out of the system. Therefore, extensions of the standard fluid model have been presented to overcome this problem. Although the proposed models do not suffer from the problem that lots come out of the system as soon as we start producing, flow times are not truly present in these models. It is not possible to determine the time it takes lots to leave once they have entered the system.

Therefore, the models presented in the previous section are (still) not satisfactory. Furthermore, according to these models any feasible throughput can be achieved by means of zero inventory. Whereas in Section 1.2 we already noticed that the nonlinear (steady state) relations depicted in Figure 1.2 should be incorporated in any reasonable model of manufacturing systems. In this section we present approximation models that do incorporate both throughput and flow time. These dynamic models are inspired by the continuum theory of highway traffic. Therefore, before presenting this dynamic model we first present some results from traffic theory.

\subsubsection{Introduction to traffic flow theory: the LWR model}

In the mid 1950s (Lighthill and Whitham 1955) and (Richards 1956) proposed a first-order fluid approximation of traffic flow dynamics. This model nowadays is known in traffic flow theory as the LWR model.

Traffic behavior for a single one-way road can be described using three variables that vary in time $t$ and space $x$ : flow $u(x, t)$, density $\rho(x, t)$ and speed $v(x, t)$. The first observation is that flow is the product of speed and density:

$$
u(x, t)=\rho(x, t) v(x, t) \quad \forall x, t .
$$

Second, for a highway without entrances or exits, the number of cars between any two locations 
$x_{1}$ and $x_{2}\left(x_{1}<x_{2}\right)$ needs to be conserved at any time $t$, i.e. the change in the number of cars between $x_{1}$ and $x_{2}$ is equal to the flow entering via $x_{1}$ minus the flow leaving via $x_{2}$ :

$$
\frac{\partial}{\partial t} \int_{x_{1}}^{x_{2}} \rho(x, t) d x=u\left(x_{1}, t\right)-u\left(x_{2}, t\right)
$$

or in differential form:

$$
\frac{\partial \rho}{\partial t}(x, t)+\frac{\partial u}{\partial x}(x, t)=0
$$

The two relations Equation 1.16 and Equation 1.17 are basic relations that any model must satisfy. As we have three variables of interest, a third relation is needed. For this third relation, several choices can be made. The LWR model assumes in addition to the relations Equation 1.16 and Equation 1.17 that the relation between flow and density observed under steady-state conditions also holds when flow and density vary with $x$ and/or $t$; i.e. for a homogeneous highway:

$$
u(x, t)=S(\rho(x, t)) .
$$

The model given by equations $1.16,1.17$, and 1.18 can predict some things encountered in traffic rather well. In order to overcome some of the deficiencies of the LWR model, in the early 1970s higher-order theories have been proposed where Equation 1.18 has been replaced by another partial differential equation, containing diffusion or viscosity terms. Unfortunately, these extended models experience some undesirable properties, as made clear in (Daganzo 1995). The most annoying of these properties is the fact that in these second-order models cars can travel backwards. Second-order models that do not suffer from this deficiency have been presented in (Jiang, Wu, and Zhu 2002; Zhang 2002).

\subsubsection{A traffic flow model for manufacturing flow}

In the previous subsection we introduced the LWR model from traffic flow theory. This model describes the dynamic behavior of cars along the highway at a macroscopic level and contains information both about the number of cars passing a certain point and about the time it takes cars to go from one point to the other. This model can not only be used for describing the flow of cars along the highway, but also for describing the flow of products through a manufacturing line. Consider, instead of a homogeneous highway, a homogeneous manufacturing line, i.e. a manufacturing line that consists of a lot of identical machines. Let $t$ denote the time and let $x$ denote the position in the manufacturing line. The behavior of lots flowing through the manufacturing line can also be described by three variables that vary with time and position: flow $u(x, t)$ measured in unit lots per unit time, density $\rho(x, t)$ measured in unit lots per unit machine and speed $v(x, t)$ measured in unit machines per unit time. Now we can relate these three variables by means of equations 1.16, 1.17 and 1.18, where in Equation 1.18 the function $S$ describes the relation between flow and density observed under steady-state conditions. To make this last statement more explicit, consider a manufacturing line consisting of $m$ machines with exponentially distributed process times and an average capacity of $\mu$ lots per unit time. Furthermore, consider a Poisson arrival process where lots arrive to the first machine with a rate of $\lambda$ lots per unit time $(\lambda<\mu)$, and assume that buffers have infinite capacity. Then we know from queueing theory (Kleinrock 1975) that the average number of lots in each workstation (consisting of a buffer and a machine) in steady state is given by

$$
N=\frac{\frac{\lambda}{\mu}}{1-\frac{\lambda}{\mu}}=\frac{\lambda}{\mu-\lambda} .
$$

In words: in steady state we have $\rho(x, t)$ is constant and

$$
\frac{1}{m} \rho(x, t)=\frac{u(x, t)}{\mu-u(x, t)},
$$

from which we can conclude that in steady state:

$$
u(x, t)=\frac{\mu \rho(x, t)}{m+\rho(x, t)} .
$$


For this example, this is the mentioned function $S(\rho)$.

With this information we can conclude that the dynamics of this manufacturing line might be described by means of the partial differential equation

$$
\frac{\partial \rho}{\partial t}+\mu \frac{\partial}{\partial x}\left(\frac{\rho}{1+\rho}\right)=0 .
$$

Together with the relations

$$
u=\frac{\mu \rho}{1+\rho} \text { and } v=\frac{u}{\rho} \text { or } v=\frac{\mu}{1+\rho}
$$

this completes our model.

Notice that contrary to the fluid models presented in the previous section, the dynamic model of Equation 1.22 is able to incorporate the stochasticity as experienced in manufacturing lines. If the manufacturing line would be in steady state, the throughput and flow time as predicted by the model of Equation 1.22 is exactly the same as those predicted by queueing theory. However, contrary to queueing theory, the model of Equation 1.22 is not a steady-state model, but also incorporates dynamics. Therefore, the model Equation 1.22 is a dynamic model that incorporates both throughput and flow time. Furthermore, given the experience in the field of fluid dynamics, the model is computationally feasible as well.

\subsection{Conclusions}

In this chapter we presented some of the models used in the modeling, analysis and control of manufacturing systems. In Section 1.3 some analytical modeling techniques and methods for analyzing steady state behavior of manufacturing systems have been introduced. To get a more detailed insight in the dynamics of a manufacturing system discrete event models, as introduced in Section 1.4 can be used. A disadvantage of discrete event models is their complexity. To reduce the complexity of discrete event models, effective proces times (EPT's) have been introduced in Section 1.5. This enables the modeling of a manufacturing system as a large queueing network.

Once the dynamics of manufacturing systems can be well described by a relatively simple discrete event model, the problem of controlling the dynamics of manufacturing systems becomes of interest. In Section 1.6 a control framework has been presented. A crucial role in this framework is played by approximation models of manufacturing systems. In Section 1.7 the most common approximation models, fluid models, have been introduced, together with some extensions of these models. These fluid models mainly focus on throughput and do not contain information on flow times. Finally, in Section 1.8, flow models have been presented that do incorporate both throughput and flow time information. 
Baker, Jr., G. A. (1965). The theory and application of the Padé approximant method. In K. A. Brueckner (Ed.), Advances in Theoretical Physics, Volume 1, pp. 1-58. New York, USA: Academic Press.

Bemporad, A., F. Borrelli, and M. Morari (2000, June). Piecewise linear optimal controllers for hybrid systems. In Proceedings of the 2000 American Control Conference, Chicago, IL, USA, pp. 1190-1194.

Bemporad, A., G. Ferrari-Trecate, and M. Morari (2000, October). Observability and controllability of piecewise affine and hybrid systems. IEEE Transactions on Automatic Control 45(10), 1864-1876.

Bemporad, A., D. Mignone, and M. Morari (1999, June). Moving horizon estimation for hybrid systems and fault detection. In Proceedings of the 1999 American Control Conference, San Diego, CA, USA, pp. 2471-2475.

Bemporad, A. and M. Morari (1999). Control of systems integrating logic, dynamics, and constraints. Automatica 35, 407-427.

Curtain, R. F. and H. Zwart (1995). An Introduction to Infinite-Dimensional Linear Systems Theory. Berlin, Germany: Springer-Verlag.

Daganzo, C. F. (1995). Requiem for second-order fluid approximations of traffic flow. Transportation Research Part B 29(4), 277-286.

DeCarlo, R. A., M. Branicky, S. Petterson, and B. Lennartson (2000). Perspectives and results on the stability and stabilizability of hybrid systems. Proceedings of the IEEE 88(7), 1069-1082.

Forrester, J. W. (1961). Industrial Dynamics. Cambridge, Massachusetts, USA: MIT Press.

Heemels, W. P. M. H., J. M. Schumacher, and S. Weiland (2000). Linear complementarity systems. SIAM Journal on Applied Mathematics 60(4), 1234-1269.

Heemels, W. P. M. H., B. d. Schutter, and A. Bemporad (2001, July). Equivalence of hybrid dynamical models. Automatica 37(7), 1085-1091.

Hopp, W. J. and M. L. Spearman (2000). Factory Physics (Second ed.). New York, USA: Irwin/McGraw-Hill.

Jacobs, J. H., P. P. v. Bakel, L. F. P. Etman, and J. E. Rooda (2005). Quantifying variability of batching equipment using effective process times. submitted to IEEE Transactions on Semiconductor Manufacturing.

Jacobs, J. H., L. F. P. Etman, E. J. J. v. Campen, and J. E. Rooda (2003, August). Characterization of the operational time variability using effective processing times. IEEE Transactions on Semiconductor Manufacturing 16(3), 511-520.

Jiang, R., Q. S. Wu, and Z. J. Zhu (2002). A new continuum model for traffic flow and numerical tests. Transportation Research. Part B, Methodological 36, 405-419.

Johansson, M. and A. Rantzer (1998, April). Computation of piece-wise quadratic Lyapunov functions for hybrid systems. IEEE Transactions on Automatic Control 43(4), 555-559.

Kingman, J. F. C. (1961). The single server queue in heavy traffic. Proceedings of the Cambridge Philosophical Society 57, 902-904.

Kleinrock, L. (1975). Queueing Systems, Volume I: Theory. John Wiley.

Kuehn, P. J. (1979). Approximate analysis of general queueing networks by decomposition. IEEE Transactions on Communication 27, 113-126.

Lighthill, M. J. and J. B. Whitham (1955). On kinematic waves. I: Flow movement in long rivers. II: A theory of traffic flow on long crowded roads. Proceedings of the Royal Society A 229, 281-345.

Little, J. D. C. (1961). A proof of the queueing formula $l=\lambda w$. Operations Research 9 , 383-387. 
Richards, P. I. (1956). Shockwaves on the highway. Operations Research 4, 42-51.

Sattler, L. (1996, November). Using queueing curve approximations in a fab to determine productivity improvements. In Proceedings of the 1996 IEEE/SEMI Advanced Semiconductor Manufacturing Conference and Workshop, Cambridge, MA, USA, pp. 140-145.

Schaft, A. J. v. d. and J. M. Schumacher (1998). Complementarity modelling of hybrid systems. IEEE Transactions on Automatic Control 43, 483-490.

Sontag, E. D. (1981, April). Nonlinear regulation: The piecewise linear approach. IEEE Transactions on Automatic Control 26(2), 346-358.

Zhang, H. M. (2002). A non-equilibrium traffic model devoid of gas-like behavior. Transportation Research. Part B, Methodological 36, 275-290. 